Classification

Physics Abstracts

$87.15-87.64 \mathrm{D}-82.80 \mathrm{P}$

\title{
Electron Energy Loss Spectroscopy Study of Iron Deposition in Human Alveolar Macrophages: Ferritin or Hemosiderin?
}

\author{
Marco Diociaiuti, Mario Falchi and Luigi Paoletti \\ Laboratorio di Ultrastrutture, Istituto Superiore di Sanità, Viale Regina Elena 299, 00161 Roma, Italy \\ (Received July 27; accepted November 9, 1994)

\begin{abstract}
Electron energy loss spectroscopy imaging (EELSI) was used to obtain Fe maps, with molecular resolution, in human alveolar macrophages. Fe concentrations were found in particular structures named siderosomes. The combined application of Extended energy loss fine structure (EXELFS) analysis, a "short-range " probe, and Selected area electron diffraction (SAED), a "long-range" probe, allowed us to investigate the atomic arrangement of proteins constituting the siderosomes. Results are compared with structural data obtained on purified proteins. It is found that the siderosomes seem to be constituted mainly by normal hemosiderins.
\end{abstract}

\section{Introduction}

Ferritin and hemosiderin are two proteins responsible for the Fe uptake in cell physiology. In particular, ferritin is composed of a protein coat of about $130 \AA$ diameter, surrounding a biomineral core of about $70 \AA$ diameter [1]. Hemosiderin is believed to be the result of the autophagic breakdown of ferritin occurring in particular membrane bounded subcellular structures (lysosomes) named "siderosomes" [2].

The atomic structural arrangement of the biomineral core of these proteins has been studied by means of X-ray Diffraction [3], Selected Area Electron Diffraction (SAED) [4]. Mossbauer Spectroscopy [5] and Exended X-ray Absorption Fine Structure analysis (EXAFS) [6]. It has been shown [1], that in both proteins, in human non pathological conditions, the biomineral core is a crystal with a structure based on $\mathrm{Fe}^{3+}$ oxide, like ferrihydrite $\left(5 \mathrm{Fe}_{2} \mathrm{O}_{3}^{*} 9 \mathrm{H}_{2} \mathrm{O}\right)$. Nevertheless, recent investigations clearly show that there are several types of biomineral cores of hemosiderins occurring in different human pathologies [7]. It is important to stress that all these structural studies were performed on bulk samples prepared using purified proteins.

In our previous study [8] we investigated the distribution of Fe particles in human Alveolar Macrophages (AM) collected from a silicotic patient. Fe particles of dimensions of the order of the ferritin core were found randomly dispersed in the cytoplasm and arranged in clusters. Such clusters are named "siderosomes" and have a high Fe concentration.

The aim of this work was the combined application of a "long-range" technique as SAED and a "short-range" technique (similar to EXAFS), such as EXELFS analysis [9] in a Transmission 
Electron Microscope (TEM) to investigate the atomic arrangement of Fe and $\mathrm{O}$ in the actual, non purified siderosome found in AM.

\section{Materials and Methods}

AM were obtained from the bronchoalveolar lavage fluids of a pottery worker exposed to silicate particles. After centrifugation, the cell pellet was fixed by the conventional glutheraldehydeosmium technique for TEM. The sample was then dehydrated in alcohol and embedded in resin (Agar 100). Sections with a thickness ranging between 900 and $500 \AA$ were cut by diamond knife, then mounted on 400 mesh copper grids and observed without the addition of contrasting media.

The Electron Energy Loss Spectroscopy Imaging (EELSI) to study the Fe distribution in the AM was performed in a dedicate TEM, the Zeiss 902, with primary electrons of $80 \mathrm{keV}$. This imaging technique is based on the energy filtering of electrons transmitted through the sample [10]. The image is realized by collecting only electrons which have lost the characteristic Fe energy loss $(708 \mathrm{eV})$.

The instrument used for the atomic structural analysis was a Philips EM430 TEM equipped with a $\mathrm{LaB}_{6}$ gun, operating at a primary beam energy of $300 \mathrm{keV}$ and equipped with a serial magnetic sector energy analyser Gatan 607 for Electron Energy Loss Spectroscopy (EELS).

SAED allows us to study the "long-range" crystalline order. After a camera length calibration performed on Au crystalline standards, diffraction patterns from an area of about $1000 \AA$ were used to identify the "long-range" crystal symmetry and to measure the lattice parameters with an error of about $3 \%$. Moreover, information concerning the diffraction domain volumes may be obtained by analysing the Debye widths. Following the Scherrer relation [11]:

$$
T-T_{\mathrm{i}} \propto 1 / d
$$

where $T$ is the Debye width, $T_{\mathrm{i}}$ the instrumental width and $d$ the diameter of the crystalline domain. Broad Debye ring widths indicate small crystalline domain volumes.

EELS spectra were acquired with counting times of $6 \mathrm{~s} / \mathrm{eV}$, acquisition angle of $16 \mathrm{mrad}$ and over areas of about $1000 \AA$ in diameter. EXELFS spectroscopy investigates the fine structure which extends for several hundred $\mathrm{eV}$ beyond an inner shell ionization edge. Analysis of this feature has demonstrated a close analogy with results obtained through the EXAFS technique [12]. The EXELFS modulation is described in terms of a $\chi(K)$ function [13] for different surrounding shells using the EXAFS formula:

$$
\chi(k)=\sum_{j} \frac{N_{j} A_{j}}{k R_{j}^{2}} \mathrm{e}^{-2 k^{2} \sigma_{j}^{2}} \mathrm{e}^{-\frac{2 R_{j}}{\lambda^{\prime}}} \sin \left(2 k R_{j}+\phi_{j}\right)
$$

where $j$ is the coordination shell of atoms surrounding the excited atom, $N_{j}$ is the coordination number of atoms located at an average distance $R_{j}$ from the central atom. The first exponential term accounts for the motion of atoms due to thermal vibration and to static displacement. The parameter $\sigma_{j}^{2}$ is the mean-square relative displacement for the $j$-th shell and the electron wave vector $k$ is related to the emitted electron energy by:

$$
k\left(A^{-1}\right)=\sqrt{0.263\left(E-E_{0}\right)(\mathrm{eV})}
$$

where $E_{0}$ is the edge onset energy.

The quantity $\lambda^{\prime}$ is a phenomenological mean free path that corresponds to a finite lifetime of the excited electron state, $A_{j}(k, \pi)$ is the backscattering amplitude of the atoms in the $j$-th shell, 

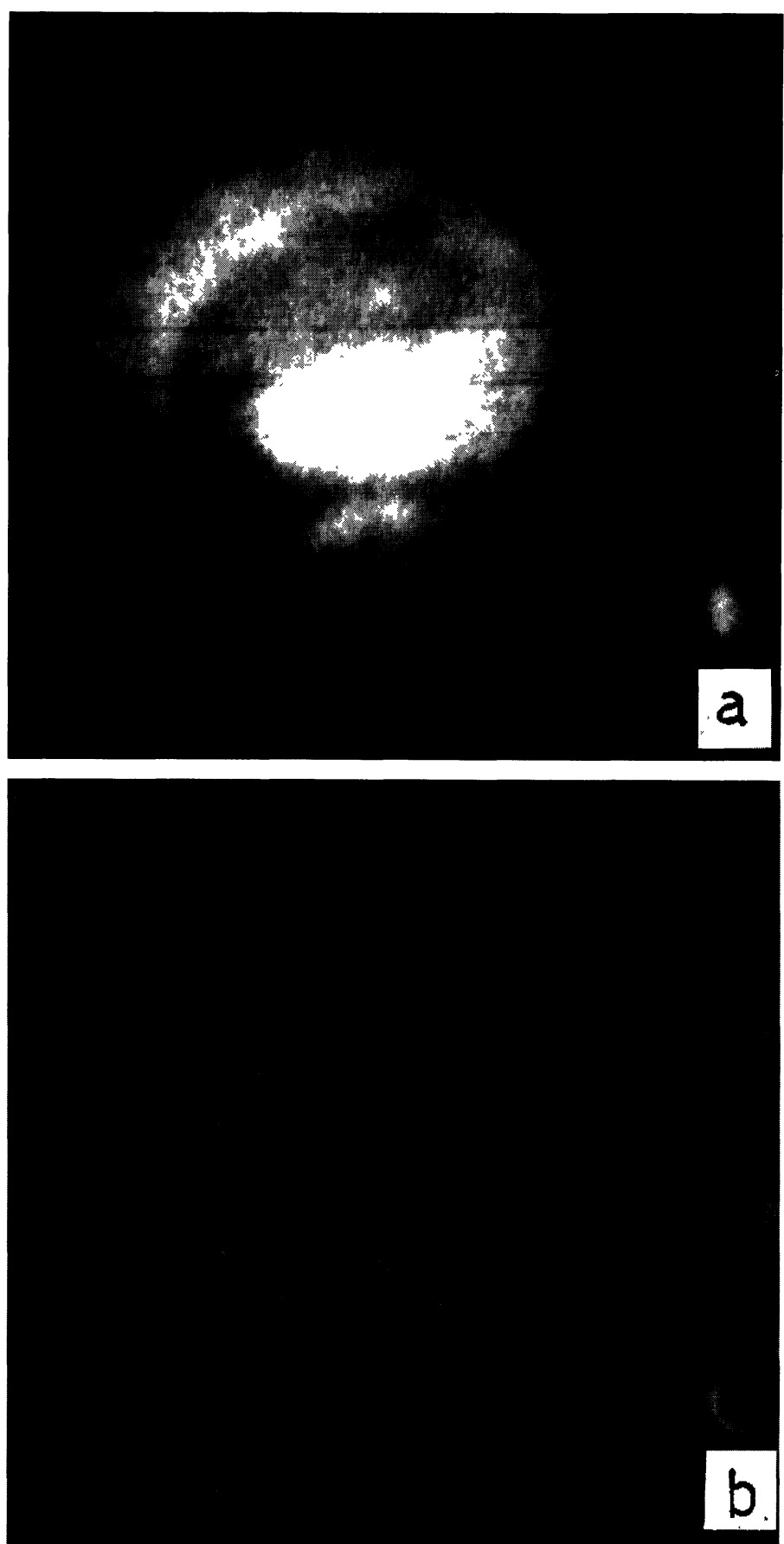

Fig. 1. - Fe EELSI a) of a typical siderosome found inside a human alveolar macrophage. b) shows the same structure in a BF image. Bar represents $2000 \AA$. 
and $\phi_{j}$ is the phase-shift experienced by the electron as it traverses the potential of the central and backscattering atoms.

The fine structures above the edges were isolated by means of an atomic background subtraction. The background was obtained by a fitting procedure of the EXELFS feature through a 3rd degree polynomial curve and subtracted from the experimental spectrum. This curve approaches the atomic background, showing no oscillating features. The EXELFS features were transformed to $k$ space using relation (3). The Radial-Distribution-Function (RDF), which gives the position of the surrounding atoms, was derived by Fourier transforming $\chi(k)$ to real space, using a wave vector range of about $2.0<k<7.0 \AA^{-1}$. EXELFS analysis was applied to $\mathrm{Fe}-L_{2,3}$ and $\mathrm{O}-\mathrm{K}$ edges located at 708 and $532 \mathrm{eV}$, respectively.

In order to verify the reliability of EXELFS analysis of $\mathrm{Fe}-L_{2,3}$ and $\mathrm{O}-\mathrm{K}$ edges, measurements were performed on standard hematite crystal. The structure of this mineral is the base of the ferrihydrite structure, that is the structure suggested for the biomineral core [1].

Finally, it must be stressed that EXELFS and SAED do not probe the same physical features, because the EXELFS technique is mainly a "short-range" probe, sensitive to three or four atomic shells, whilst SAED is sensitive mainly to "long-range" order effects [14].

\section{Results and Discussion}

Fe EELSI of a typical siderosome in AM is shown in Figure 1a. In the same Figure $1 \mathrm{~b}$ we show the same object observed in Bright Field. It is evident that dark zones in BF correspond to Fe (white zones) in the EELSI.

Figure 2 shows EELSI (a) and BF (b) at higher magnification of a zone of the same siderosome where proteins are arranged in a linear array, probably because of the interaction with a cellular membrane.

A SAED pattern obtained from the same siderosome is reported in Figure 3. Only three Debye rings are clear but another two can be distinguished, the wider one like a band. The main ring measurements lead to lattice spacings of $2.44 \pm 0.07,2.16 \pm 0.06$ and $1.67 \pm 0.05 \AA$, the other two to $1.92 \pm 0.06$ and $1.43 \pm 0.04 \AA$. These values are in agreement, within the experimental error, with those reported [4] for hemosiderin and ferritin, corresponding to the well ordered ferrihydrite structure. All the rings are characterized by a width broader than the instrumental width measured on the reference Au standard. In particular the last ring seems to be wider than others. The Debye ring width is an estimation of the dimension of the crystalline domain and broad rings are due to small domains.

These observations suggest that the crystal arrangement of the biomineral cores within the siderosome is based on homogeneous domains of about $60 \AA$ diameter with the ferrihydrite structure, as suggested for purified hemosiderin [4].

In such structure a local probe like EXELFS analysis can be very useful.

In Figure 4, $\mathrm{Fe}-L_{2,3}$ (a) and O-K (c) EXELFS oscillations relative to the standard hematite sample are shown. In Figures $4 \mathrm{~b}$ and $4 \mathrm{~d}$ the relative RDFs are reported. Black bars in the RDFs indicate the $\mathrm{Fe}-\mathrm{O}, \mathrm{Fe}-\mathrm{Fe}, \mathrm{O}-\mathrm{O}$ distances relative to the hematite structure [15]. The differences between experimental and theoretical distances are due to the phase-shift correction to be made in the EXELFS analysis. The correction can be calculated using the spherical-wave tabulated phase functions for $\mathrm{Fe}-L_{2,3}$ and $\mathrm{O}-\mathrm{K}$ edges [16]. We obtained similar values around $0.22 \AA$ (variations in the second figure) for all corrections.

All peaks in both RDFs of hematite (4b) and (4d) are in good agreement, after the phase-shift correction, with theoretical values. This result clearly demonstrates the ability of EXELFS to study the atomic arrangement at the local level. 

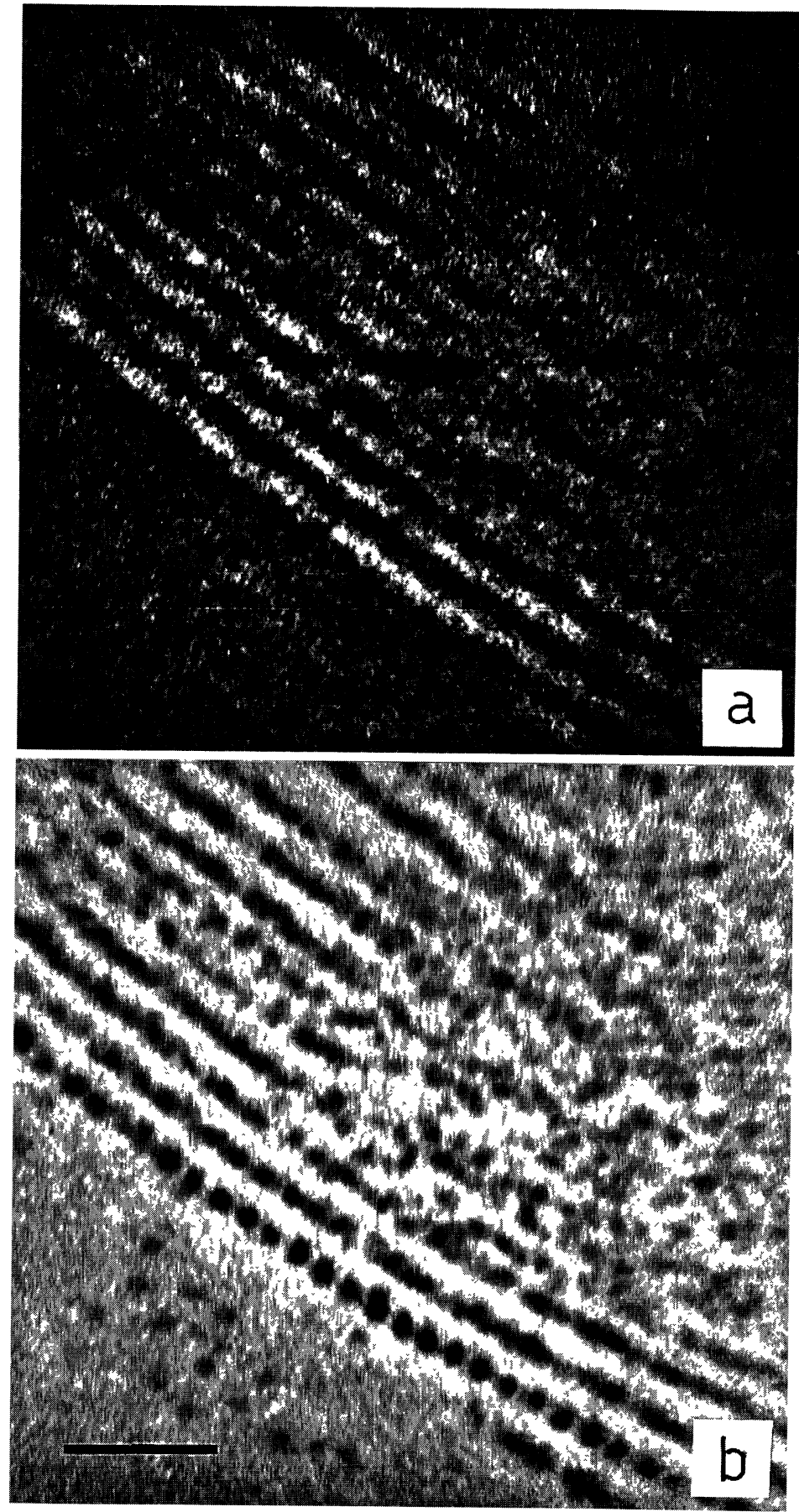

Fig. 2. - Fe EELSI a) and BF b) images of a particular ordered structure of the siderosome, probably due to the interaction of proteins with cellular membranes. Bar represents $400 \AA$. 


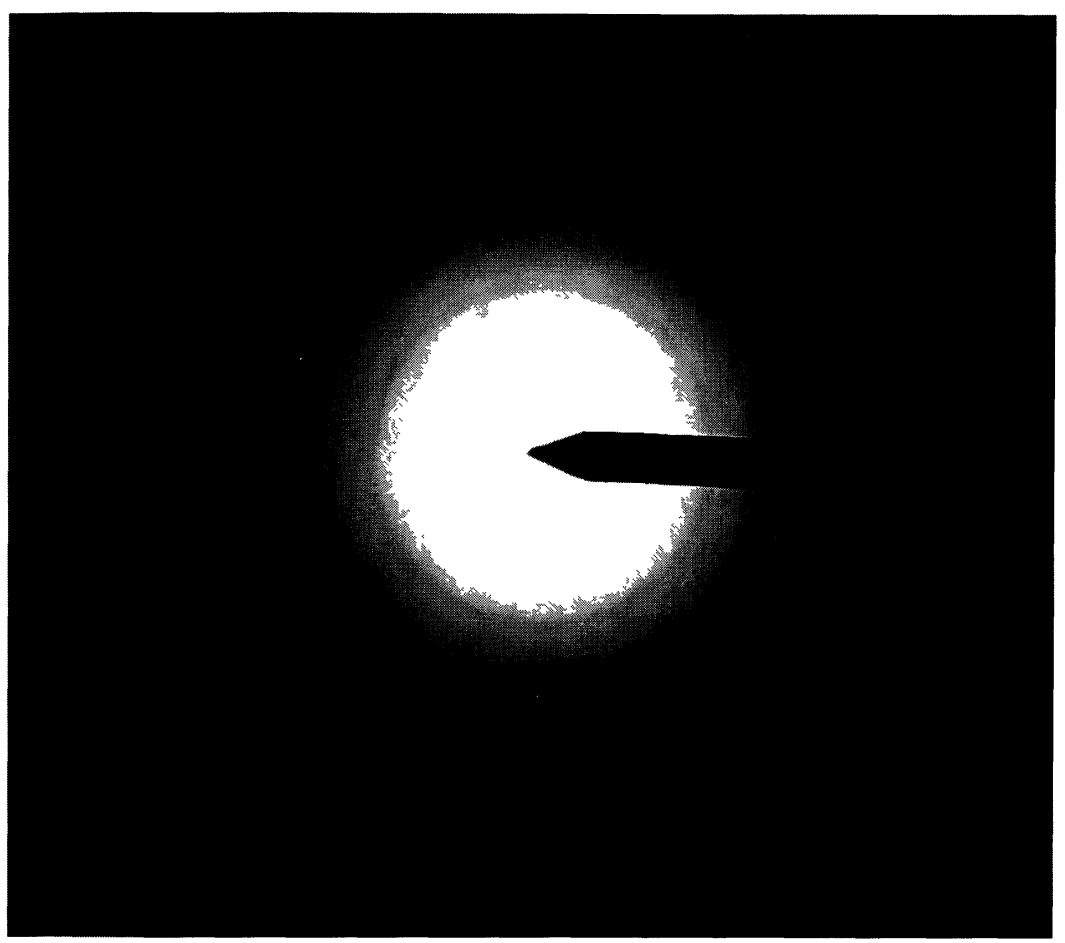

Fig. 3. - SAED pattern from a wide area $(1000 \AA)$ of the siderosome.

The same EXELFS analysis was performed on the siderosome of Figure 1. The results are summarized in Figure 5. The Fe RDF (5b) is quite similar to that of hematite (4b). The first coordination shells $\mathrm{Fe}-\mathrm{O}$ and $\mathrm{Fe}-\mathrm{Fe}$ are distinguishable, even if the $\mathrm{Fe}-\mathrm{Fe}$ peak of hematite seems to be better resolved. From this RDF it can be stated that the local environment of $\mathrm{Fe}$ in the siderosome is very similar to that of the hematite structure, that is that of ferrihydrite. Both bond lengths determined by EXELFS and lattice spacings measured by SAED are typical of the hematite/ferrihydrite structure [15].

The O RDF (5d) for the siderosome is quite different from that of hematite. The first peak is the more intense and is located at $1.5 \AA$ against $1.7 \AA$ in the hematite. The second peak has the location of the hematite $(2.6 \AA)$ but is less intense. This discrepancy can be explained by noting that in the siderosome $\mathrm{O}$ is present inside the biomineral core, probably coordinated with $\mathrm{Fe}$ as in the hematite and, outside the core, in the protein coat coordinated with $\mathrm{C}$. This short $\mathrm{O}-\mathrm{C}$ double bond would mask the O-Fe peak, with the result shown in Figure $5 \mathrm{~d}$ but the strong coordination O-O (2.6 $\AA)$, characteristic of the hematite/ferrihydrite structure, is still evident in the siderosome RDF of Figure $5 d$.

\section{Conclusion}

EELSI has allowed us to study the molecular arrangement of proteins storing Fe inside the actual AM. The combined application of a structural "long-range" probe, as SAED, and a "short-range" probe, as EXELFS, has given us information about the atomic arrangement of the biomineral 


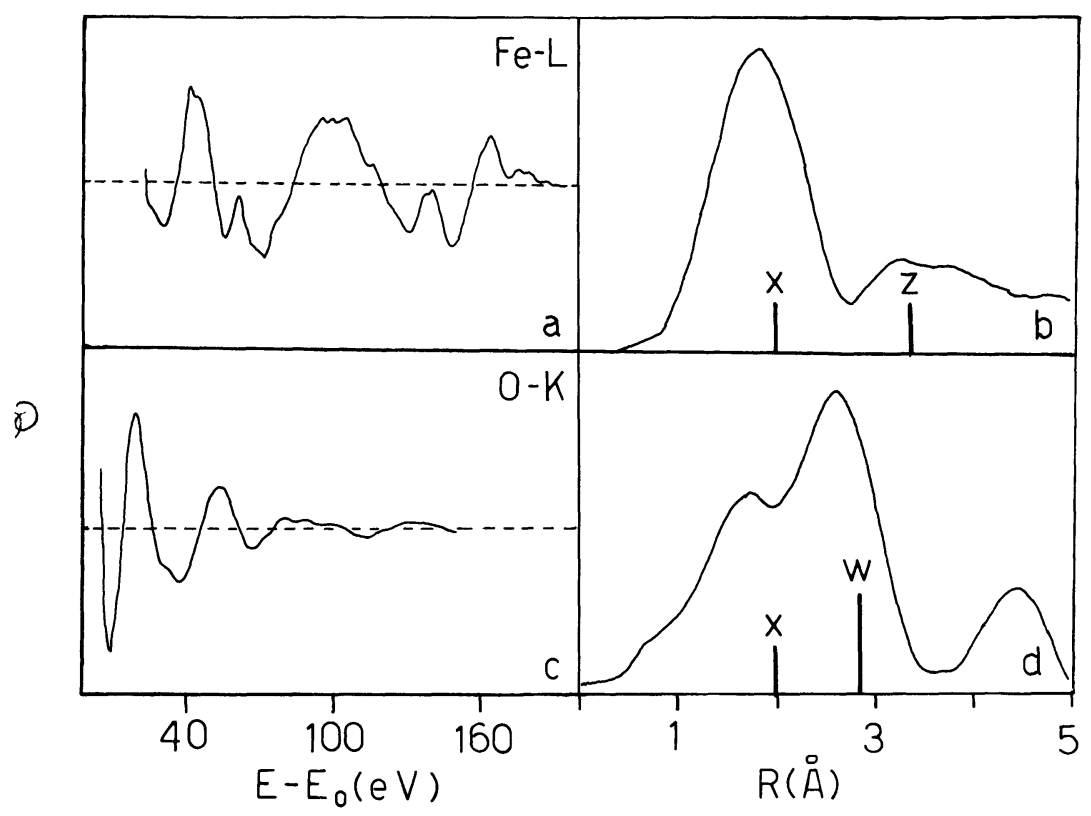

Fig. 4. - EXELFS oscillations extracted from $\mathrm{Fe}-L_{2,3}$ a) and $\mathrm{O}-\mathrm{K} \mathrm{c}$ ) edges in a spectrum obtained from standard hematite. The relative RDFs are shown $b, d)$ together with black bars representing the major $\mathrm{Fe}-\mathrm{O}(x), \mathrm{Fe}-\mathrm{Fe}(z)$ and $\mathrm{O}-\mathrm{O}(w)$ coordination distances in the hematite.

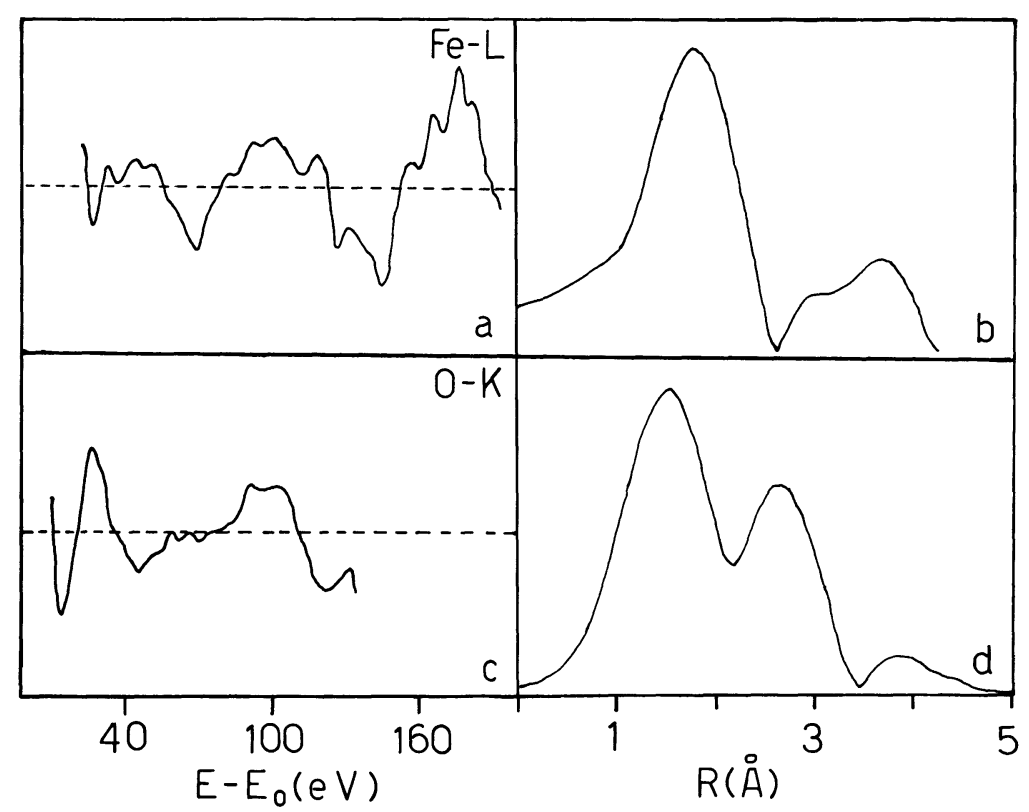

Fig. 5. - EXELFS oscillations from $\mathrm{Fe}-L_{2,3}$ a) and $\mathrm{O}-\mathrm{K} \mathrm{c}$ ) with the corresponding RDFs b, d) for the actual siderosome. 
core of proteins constituting the siderosomes. There is a good agreement between SAED and EXELFS structural results. Both probes suggest a crystalline structure based on the hematite/ ferrihydrite, proposed for both purified human ferritin and normal hemosiderin.

We conclude that siderosomes found in the AM of a silicotic patient are constituted by hemosiderin with a biomineral core of structure similar to the biomineral core of ferritin.

We want to stress that this study has been realised on actual AM, collected in vivo, where proteins were not purified and were in their actual biological environment. Moreover, that for the first time, the EXELFS analysis has been successfully performed on a biological sample, furnishing structural information comparable to EXAFS.

\section{References}

[1] Massover W.H., Micron 24/4 (1993) 389.

[2] Andrew C., Treffry A., Harrison M., Biochem. J. 245 (1987) 439.

[3] Alix D., Girardet J.-L., Lawrence J.-J., Mouriquand C., J. Microsc. Paris 12 (1971) 33.

[4] Dickson D.P.E., Reid N.M.K., Mann S., Wade V.J., Ward R.J., Peters T.J., Biochem. Biophys. Acta 957 (1988) 81.

[5] Bauminger E.R., Harrison P.M., Heschel D., Nowik I., Treffry A., Biochem. Biophys. Acta 1118 (1991) 48.

[6] Mackle P., Garner C.D., Ward R.J., Peters T.J., Biochem. Biophys. Acta 1115 (1991) 145.

[7] Ward R.J., Ramsey M.H., Dickson P.D.E., Florence A., Crichton R.R., Peters T.J., Mann S., Eur. J. Biochem. 209 (1992) 847.

[8] Diociaiuti M., Falchi M., Paoletti L., Mikrochim. Acta 114/115 (1994) 285.

[9] Filipponi A., Lozzi L., Passacantando M., Picozzi P., Santucci S., Diociaiuti M., Phys. Rev. B 47/14 (1993) 8494.

[10] Egerton R.F., Electron Energy Loss Spectroscopy in the Electron Microscope (Plenum Press, New York 1986).

[11] Heidenreich R.D., Fundamentals of Transmission Electron Microscopy (Wiley-Interscience, New York 1972).

[12] Teo B.K. and Joy D.C., EXAFS Spectroscopy Techniques and Applications (Plenum Press, New York 1981).

[13] Leapman R.D., Grunes L.A., Fejes P.L. and Silcox J., in: EXAFS Spectroscopy, Techniques and Applications, B.K. Teo and D.C. Joy Eds. (Plenum Press, New York 1981) p. 217.

[14] Diociaiuti M., Picozzi P., Santucci S., Lozzi L., De Crescenzi M., J. Microsc. Oxford 166 (1992) 231.

[15] Blake R.L., Essevick R.E., Zoltai T., Finger L.W., Am. Mineral.51 (1960) 123.

[16] Mckale A.G., Veal B.W., Paulinkas A.P., Chan S.K. and Knapp G.S., J. Am. Chem. Soc. 110 (1988) 376. 